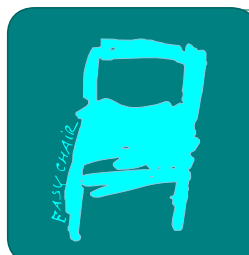

Kalpa Publications in Computing

Volume 3, 2017, Pages 55-63

RV-CuBES 2017. An International Workshop on

Competitions, Usability, Benchmarks, Evaluation, and Standardisation for Runtime Verification Tools

\title{
Runtime Verification using LARVA
}

\author{
Christian Colombo and Gordon J. Pace
}

Department of Computer Science, University of Malta, Malta.

\begin{abstract}
LARVA, which has been in use and continuous development for almost a decade, has been extended in several ways and used in a wide range of scenarios, from industrial deployment to educational ones. In this paper we give an overview of the runtime verification tool LARVA and its extensions and uses.
\end{abstract}

\section{Introduction}

LARVA $[17,18]$ is a Java-based runtime verification tool, originally developed by the authors between 2007 and 2008 with the main drive being that of an industrial financial transaction system guiding the choice of features and design of the tool. Since then, the tool has been used (i) in various industrial projects and case studies; (ii) as a test-bed (to experiment with extensions and re-implementations) for research purposes; (iii) for teaching purposes. In the rest of the paper, after an overview of LARVA in the next section, we dedicate a section to each of these three aspects. Finally, we relate LARVA to other tools and conclude.

\section{High-Level Overview of the Tool}

Perhaps the most defining aspect of LARVA is the use of symbolic automata as the basis of the specification language. This allows users who are already familiar with finite state machines to quickly grasp how to specify properties, while at the same time ensuring Turing completeness by allowing Java code to be embedded in the transitions. As an example consider the property below which keeps track of a risk value depending on a sequence of transaction actions carried out by the user. In particular note how by detecting relevant events, the automaton transitions from one state to another and updates a float value, risk, in the process. The label of the transition (given in square brackets) contains three backslash separated elements - the event which triggers the transition, a condition which must hold for the transition to be taken and the action to be executed when the transition is taken. Note that transitions are processed in the order in which they appear to avoid non-determinism ${ }^{1}$.

Variables \{float risk;

\footnotetext{
${ }^{1}$ In the examples given in the paper, we give snippets of LARVA scripts, with parts not directly relevant to the point being illustrated being left out. For examples of full scripts, the reader is referred to the user manual ${ }^{2}$ and examples provided with LARVA.
}

G. Reger and K. Havelund (eds.), RV-CuBES 2017 (Kalpa Publications in Computing, vol. 3), pp. 55-63 


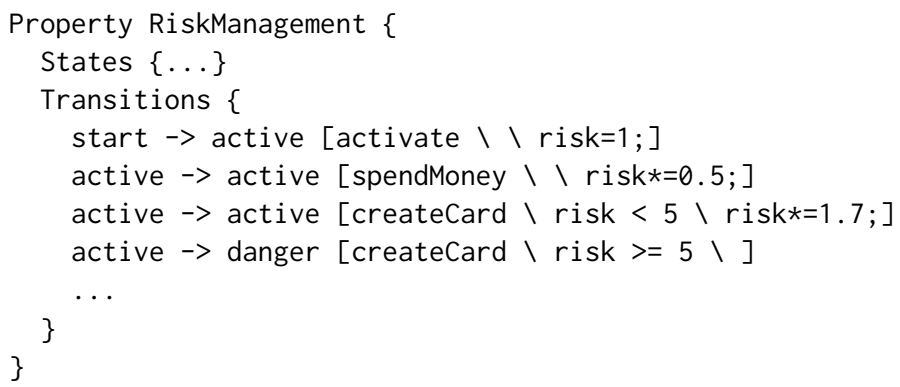

Another defining element of LARvA is the foreach construct which allows top level universal quantification in specifications in a straightforward manner. Building on the previous example, using the foreach construct, the property can be instantiated for every unique user encountered $^{3}$ :

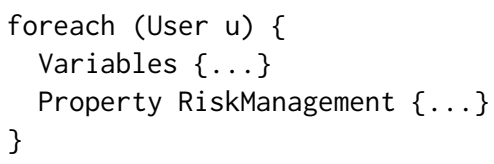

LARVA also provides native timers, which can be used to trigger or guard transitions, making it easier to define real-time properties such as marking a user inactive following 30 days without carrying out any transaction (and then detecting a violation if any transaction is detected while inactive):

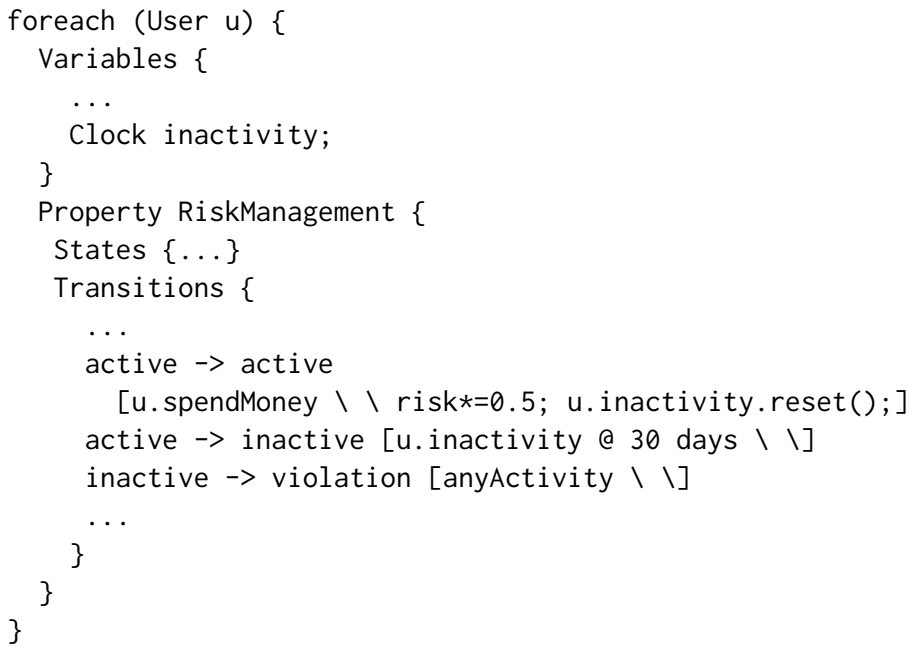

Finally, to facilitate the definition of properties which can benefit from a modular definition, LARVA properties can communicate between themselves through the use of non-blocking channels - allowing the transmission of any Java object across monitors over these internal communication events. In what follows, we extend the example such that the individual user's property sends updates to a central property which keeps count of high risk users:

\footnotetext{
${ }^{3}$ This effectively checks the property for every instance of class User using the default notion of object equivalence, although which notion of equivalence to use can be set by the property writer.
} 


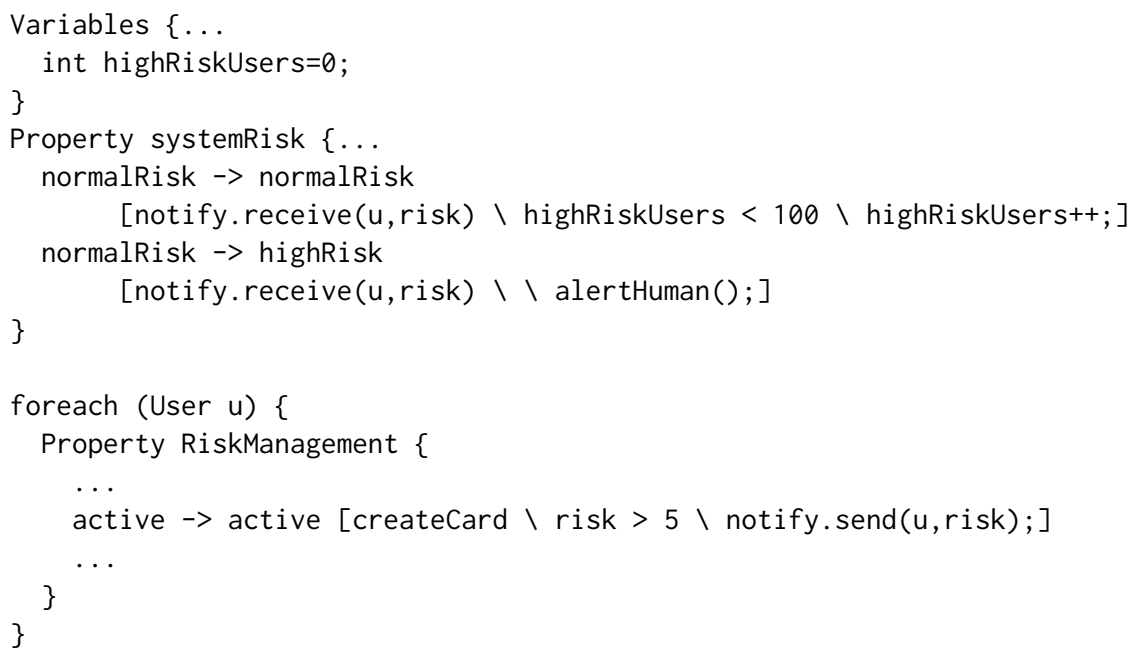

Once the specification is defined as in the above snippets, the user associates events with Java operations such as a method call entry or exit, exception throw, etc. Subsequently, through the use of aspect-oriented technology [22], the compilation process links the code points referred to by the events to monitoring code which essentially consists of a series of if-then-else statements corresponding to the transitions.

To support users in making the best use of the tool, LARVA is supported by a comprehensive user manual providing a running example, ships with a number of sample scripts and systems ${ }^{4}$, and has also been more recently integrated into an Eclipse plugin ${ }^{5}$ which provides syntax highlighting and automatic seamless generation of the monitoring (.lrv) files in an Eclipse project.

\section{Case Studies}

LARVA was originally conceived in the context of an industrial case study [17] in the financial transactions industry, and it has since been (extended and) applied to other case studies in this domain [14] (see Section 4). However, LARVA has also been applied to numerous other case studies from other domains including astronomy, user profiling, business intelligence, and video surveillance. To avoid repeating what has already been published, below we only give a short description:

Business intelligence through Facebook Keeping track of all the relevant comments, messages, and posts might be a daunting task for a marketing officer responsible for social media. Through the use of runtime monitoring this can be alleviated by enabling the user to specify rules in a purposely-designed language [13, 16].

Profiling user web interfaces Understanding how users use a web interface can be crucial in improving the design to help users complete their tasks efficiently. Through the use of runtime verification, voluminous logs of user actions on a web interface could be recognised efficiently as fitting into particular usage patterns and generating usage statistics [16].

\footnotetext{
${ }^{4}$ LARVA code, manual, and samples are available on https://github. com/ccol002/larva-rv-tool

${ }^{5}$ http://www.cs.um.edu.mt/svrg/Tools/LARVA/update-site/
} 
Adaptive user interfaces User interfaces which adapt to the user's behaviour are known to be notoriously difficult to develop and maintain. LARVA was used to instrument such adaptive features using a monitor oriented programming approach, and evaluation of (i) how developers fare when using such an approach as opposed to instrumenting the code directly; and (ii) a software metric-based complexity comparative analysis of the two approaches was conducted [6].

Tax fraud detection Tax fraud experts would typically rely on technical personnel to carry out their querying of the data available. To avoid the communication overhead, with its hurdles of misunderstandings, a controlled natural language which automatically compiles into monitors has been designed and implemented [7].

Intelligent video surveillance Watching and analysing hours of surveillance footage is tedious for humans to do. One way of automating this is by allowing a user to specify rules which would classify suspicious behaviour. A case study has been carried out at a high security venue in Malta using this technique [16].

Astronomy Radio telescope observations result in large chunks of data which needs to be sifted for patterns of interest. While there are techniques in place to achieve this, runtime monitoring techniques provide another alternative [16].

Monitoring an enterprise service bus In component-based systems such as an enterprise service bus, where components can easily be added and removed, runtime monitoring provides more benefit over and above testing (than in the case of monolithic systems), since it is harder for testing to be representative all the possible environments a component would be functioning in. As a means of exploring ways in which runtime verification could be applied in this context, LARVA has been instrumented in several ways together with the Mule Enterprise Service Bus [10].

Network intrusion detection Intrusion detection systems often rely on a number of rules which their users set to identify suspicious behaviour. A case study was carried out using duration calculus as a specification language, which was then translated automatically into native LARVA automata [20].

The case studies reviewed above were all successful in the sense that we managed to define and monitor non-trivial properties according to the context. If one considers a tougher measure of success, whether LARva remained in use in an industrial system following the case study, then only "profiling user web interfaces" passes the test.

\section{Variations of LARVA}

Over the years, a number of extensions have been added to LARva with the aim of providing more features and make it more easily usable. We split this into different types of extensions: (i) extensions from a notational convenience point of view, i.e., providing additional notations in which to express properties (but not extended expressivity per se), and (ii) architectural modifications which enable LARVA to provide additional features.

\section{Notational convenience}

Interval time logics While timer actions offer the property engineer a quick way of constructing monitors for real-time properties dealing with points in time, reasoning about time 
intervals might be more challenging. For example consider the property "There should never be more than three bad logins in any one minute interval". While it can be encoded using timers, it would be significantly straight forward had the notion of an interval been natively supported. In this respect, a LARVA add-on ${ }^{6}$ provides conversion of a subset of duration calculus formulae [20] as well as QDDC formulae [19] into LARVA notation.

Statistics Taking the view of monitor-oriented programming instead of strict runtime verification, we explored the possibility of using monitors to collect statistical data. In this respect, we created a notation extension [12] which supports two main additional constructs: one supporting point statistics and one supporting interval statistics. Point statistics are those which aggregate statistical results over the whole system history while interval statistics compute information over intervals defined in terms of the starting and ending events.

Domain specific languages LARVA has also been used as an intermediate language to which domain specific languages can be compiled. Of particular significance are the following two: Firstly, we have compiled a language in the domain of business intelligence gathering from social media [13]. Secondly, we have compiled a language from the domain of tax fraud detection into LARVA specifications [7].

\section{Feature enhancement}

Asynchronous monitoring Where one would want to keep the intrusion of monitoring on the system to a bare minimum, an option is to monitor asynchronously, i.e., allowing the monitor to lag behind the system. A version of LARVA does this by consuming events from a database rather than aspect-oriented programming [14].

Database support When monitoring real-life industrial system, a considerable concern is to ensure the monitor behaves correctly even under a system crash, or when the resources required for monitoring grow significantly large. For this purpose, a version of LARVA comes equipped with a database to store and retrieve monitors and their state [14]. This means that in case of a system crash, the monitor state would not be lost, and the size of the monitor state would not be constrained by the main memory. Naturally this comes at the additional cost of database reads and writes. At the time of writing, this process is not optimised (e.g., via caching) and therefore every monitor access a database read, while a monitor state update costs a database write.

Monitor fast-forwarding Due to the monitors usually needing to keep some kind of state (essentially, a summary of the system's history), if monitors are substantially modified or new ones introduced, the monitor state might have to be rebuilt - taking into consideration all of the system's history. One way of bypassing this is if the property writer can provide a way of directly abstracting away irrelevant details from the system's history to compute the monitor state at a particular point in time without replaying all the events individually, i.e., the point at which the new (or modified) monitors are introduced. For example, instead of computing the status of a user by going through all the transactions from the beginning, the latest transactions are used to deduce the status - with the assumption that the system up to a certain point was working correctly. This has been incorporated as a feature in one of the flavours of LARVA [15] where the user is allowed to specify event abstraction code.

\footnotetext{
${ }^{6}$ Available in the LARva repository.
} 
Dynamic state generation In cases where the number of explicit monitor states is significantly high, the property engineer might prefer to encode such states programmatically, i.e., compute the next state program without providing an enumeration of all the possible states (similar to property monitoring through rewriting as opposed to full a priori state space exploration). For example consider using LARva to monitor a regular expression: one way would be to create a state for every possible evolution of the expression; another way is to provide the algorithm which generates regular expression evolutions, i.e., monitoring states, on the fly. The tool adaptation supporting this feature has been called dLARVA [9].

Memory-bounded monitoring A major concern in monitoring is the resources used, since these are typically consumed from those available to the system. While this is virtually impossible to avoid, one way an engineer could control the effects this might have on the system is by being aware of a predetermined upperbound so that enough resources can be allocated by design. This is supported in LARVA [19] by enabling monitors to be defined as Lustre [8] code for which computing strict resource consumption upperbounds is standard.

Combining LARVA with data-flow static analysis Overheads induced by runtime verification can be a concern in some systems. One way in which this has been addressed in the literature is by using static analysis to simplify the properties and reduce what still has to be checked at runtime. In STaRVOOrs ${ }^{7}[1,2]$, LARVA has been combined with the deductive verification tool $\mathrm{KeY}$ to verify properties which combine data-flow aspects (in the form of pre-/postconditions) and control-flow ones (in terms of LARvA automata).

Combining LARVA with control-flow static analysis A complementary approach to reducing properties based on data-flow static analysis, is that of using control-flow analysis. In CLarva [3], techniques originally developed for Clara [5] were extended for the symbolic automata properties used by LARVA, allowing for reduction of instrumentation points and property complexity, thus reducing monitoring overheads.

We conclude this section by noting that while the main LARvA implementation is for Java (and all the above extensions are in Java), there are also basic implementations for Erlang [11], $\mathrm{C} \#$, and an adaptation for the (Java-based) OSGi framework [21].

\section{LARVA in Education}

Over the years, LARva has been used for teaching runtime verification. Due to its automatabased notation, even undergraduate students with a knowledge of Java and automata can use the tool. Students are initiated by being given two sample systems and scripts (two of those provided with LARVA downloads), and a set of easy exercises modifying these scripts. Typically after this tutorial, lasting around an hour, students are then able to continue exploring the advanced features of the tool through the user manual. Subsequently, they are given a more substantial case study (e.g., elevator system) accompanied with a specification written in natural language, and they are expected to monitor it using the LARVA. The students manage to complete such an assignment with approximately four hours of introduction to runtime verification, using LARVA as an example tool, and the one-hour tutorial described above.

${ }^{7}$ http://cse-212294.cse. chalmers.se/starvoors/ 
To a lesser extent, the tool has also been used in graduate courses. In this case, students would (with some exceptions) have already used LARVA in their undergraduate course. In the graduate course, students get to build their own simplified version of LARVA. They are given basic infrastructure code (for instance to parse properties), and through the course they add code to enable their system to mimic LARVA features. To achieve this, students are given around 14 hours of lectures which dovetail with their practical work (at home) on each feature of the tool (e.g., integration of aspect-oriented programming, support for finite state automata, and the use of regular expressions).

\section{Related Work and Conclusions}

There are several tools which are close to LARVA in both their architecture and purpose, particularly, JavaMOP [23] (which came before LARVA) and MarQ [24] (which came after). What might be considered as the contribution of body of work surrounding LARVA is that it broke off from the traditional specification languages (particularly LTL) towards an automaton-based notation. Another interesting difference is that LARVA has always been used on case studies significantly different from what previous Java tools had been applied to: Before LARVA, Java properties revolved around correct API usage checks (e.g., correct action sequences on iterators [4]); on the other hand, the properties LARva has been used for, are higher level "business rules" such as the one in the example above. The philosophy behind this choice was that if the Java API requires monitoring, then this should be something which ships with the JVM (which can perhaps be turned on and off), without involving the programmer in such concerns. Conversely, having a specification language which is high-level enough could even allow the quality assurance personnel to write the properties rather than the developers. This has the added benefit that the property writers are not the programmers - with more value for one view of the system validating the other.

While the ease with which one can write properties would be one of the pluses of LARVA, it is not a tool which has been primarily designed for efficiency ${ }^{8}$. However, typically when monitoring high level business rules, one would not expect events of interest to occur as frequently as when monitoring low level properties. Another element which has never been properly tackled in LARVA is dealing with concurrency (and distribution). Although LARVA ensures there are no data races by strictly avoiding non-determinism, the extremely prudent approach of serialising all threads leads to inefficiency.

Summary Summarising the above into the main characteristics of LARVA:

+ Ideal where high property expressivity is preferred.

+ Suitable for high-level properties.

+ Low learning curve for non-logicians.

- Not for applications where efficiency is crucial.

- No support for dealing with concurrency or distribution efficiently.

\footnotetext{
${ }^{8}$ While LARVA uses hashmaps to enable quick monitor lookup, the implementation of more advanced features such as those involving nested hashmaps are not optimised.
} 


\section{References}

[1] Wolfgang Ahrendt, Jesús Mauricio Chimento, Gordon J. Pace, and Gerardo Schneider. A specification language for static and runtime verification of data and control properties. In FM 2015: Formal Methods - 20th International Symposium, Oslo, Norway, June 24-26, 2015, Proceedings, pages 108-125, 2015.

[2] Wolfgang Ahrendt, Jesús Mauricio Chimento, Gordon J. Pace, and Gerardo Schneider. Verifying data- and control-oriented properties combining static and runtime verification: theory and tools. Formal Methods in System Design, pages 1-66, 2017.

[3] Shaun Azzopardi, Christian Colombo, and Gordon J. Pace. Control-flow residual analysis for symbolic automata. In Proceedings Second International Workshop on Pre-and Post-Deployment Verification Techniques, PrePost@iFM 2017, Torino, Italy, 19 September 201\%., pages 29-43, 2017.

[4] Eric Bodden, Feng Chen, and Grigore Rosu. Dependent advice: a general approach to optimizing history-based aspects. In Aspect-oriented software development (AOSD), pages 3-14. ACM, 2009.

[5] Eric Bodden, Patrick Lam, and Laurie J. Hendren. Clara: A framework for partially evaluating finite-state runtime monitors ahead of time. In Runtime Verification - First International Conference, RV 2010, St. Julians, Malta, November 1-4, 2010. Proceedings, pages 183-197, 2010.

[6] Aaron John Buhagiar, Gordon J. Pace, and Jean-Paul Ebejer. Engineering adaptive user interfaces using monitoring-oriented programming. In 2017 IEEE International Conference on Software Quality, Reliability and Security, QRS 2017, Prague, Czech Republic, July 25-29, 2017, pages 200-207, 2017.

[7] Aaron Calafato, Christian Colombo, and Gordon J. Pace. A controlled natural language for tax fraud detection. In Controlled Natural Language - 5th International Workshop, CNL 2016, Aberdeen, UK, July 25-27, 2016, Proceedings, pages 1-12, 2016.

[8] Paul Caspi, Daniel Pilaud, Nicolas Halbwachs, and John Plaice. Lustre: A declarative language for programming synchronous systems. In Conference Record of the Fourteenth Annual ACM Symposium on Principles of Programming Languages, Munich, Germany, January 21-23, 1987, pages 178-188, 1987.

[9] John Paul Cassar, Christian Colombo, and Gordon J. Pace. Dynamic automata in larva. Technical Report 02-WICT-2010, Department of Computer Science, University of Malta, 2010.

[10] Christian Colombo, Gabriel Dimech, and Adrian Francalanza. Investigating instrumentation techniques for ESB runtime verification. In Software Engineering and Formal Methods - 13th International Conference, SEFM 2015, York, UK, September 7-11, 2015. Proceedings, pages 99-107, 2015.

[11] Christian Colombo, Adrian Francalanza, and Rudolph Gatt. Elarva: A monitoring tool for erlang. In Runtime Verification - Second International Conference, (RV), volume 7186 of Lecture Notes in Computer Science, pages 370-374. Springer, 2012.

[12] Christian Colombo, Andrew Gauci, and Gordon J. Pace. Larvastat: Monitoring of statistical properties. In Runtime Verification - First International Conference, (RV), volume 6418 of Lecture Notes in Computer Science, pages 480-484. Springer, 2010.

[13] Christian Colombo, Jean-Paul Grech, and Gordon J. Pace. A controlled natural language for business intelligence monitoring. In Natural Language Processing and Information Systems - 20th International Conference on Applications of Natural Language to Information Systems, NLDB 2015 Passau, Germany, June 17-19, 2015 Proceedings, pages 300-306, 2015.

[14] Christian Colombo, Gordon Pace, and Patrick Abela. Safer asynchronous runtime monitoring using compensations. Formal Methods in System Design, 41(3):269-294, 2012.

[15] Christian Colombo and Gordon J. Pace. Fast-forward runtime monitoring - an industrial case study. In Runtime Verification, Third International Conference, RV 2012, volume 7687 of Lecture Notes in Computer Science, pages 214-228. Springer, 2012.

[16] Christian Colombo, Gordon J. Pace, Luke Camilleri, Claire Dimech, Reuben A. Farrugia, Jean- 
Paul Grech, Alessio Magro, Andrew C. Sammut, and Kristian Zarb Adami. Runtime verification for stream processing applications. In Leveraging Applications of Formal Methods, Verification and Validation: Discussion, Dissemination, Applications - 7th International Symposium, ISoLA 2016, Imperial, Corfu, Greece, volume 9953 of Lecture Notes in Computer Science, pages 400-406, 2016.

[17] Christian Colombo, Gordon J. Pace, and Gerardo Schneider. Dynamic event-based runtime monitoring of real-time and contextual properties. In Formal Methods for Industrial Critical Systems (FMICS), volume 5596 of Lecture Notes in Computer Science, pages 135-149. Springer, 2008.

[18] Christian Colombo, Gordon J. Pace, and Gerardo Schneider. Larva - safer monitoring of realtime java programs (tool paper). In Software Engineering and Formal Methods (SEFM), pages 33-37. IEEE, 2009.

[19] Christian Colombo, Gordon J. Pace, and Gerardo Schneider. Resource-bounded runtime verification of java programs with real-time properties. Technical Report CS2009-01, Department of Computer Science, University of Malta, 2009. Available from http://www. cs. um. edu.mt/ reports.

[20] Christian Colombo, Gordon J. Pace, and Gerardo Schneider. Safe runtime verification of real-time properties. In Formal Modeling and Analysis of Timed Systems, 7th International Conference (FORMATS), volume 5813 of Lecture Notes in Computer Science, pages 103-117, Budapest, Hungary, 2009.

[21] Yufang Dan, Nicolas Stouls, Stéphane Frénot, and Christian Colombo. A Monitoring Approach for Dynamic Service-Oriented Architecture Systems. In SERVICE COMPUTATION 2012: The Fourth International Conferences on Advanced Service Computing, pages 20-23. XPS (Xpert Publishing Services), 2012.

[22] Gregor Kiczales, John Lamping, Anurag Mendhekar, Chris Maeda, Cristina Lopes, Jean-Marc Loingtier, and John Irwin. Aspect-oriented programming, pages 220-242. Springer Berlin Heidelberg, Berlin, Heidelberg, 1997.

[23] Patrick O’Neil Meredith, Dongyun Jin, Dennis Griffith, Feng Chen, and Grigore Roşu. An overview of the MOP runtime verification framework. International Journal on Software Techniques for Technology Transfer, 14:249-289, 2012.

[24] Giles Reger, Helena Cuenca Cruz, and David E. Rydeheard. Marq: Monitoring at runtime with QEA. In Tools and Algorithms for the Construction and Analysis of Systems TACAS, volume 9035 of Lecture Notes in Computer Science, pages 596-610. Springer, 2015. 